\title{
Micro-Raman Measurement of Bending Stresses in Micromachined Silicon Flexures
}

\author{
V. T. Srikar, Anna K. Swan, M. Selim Ünlü, Senior Member, IEEE, Bennett B. Goldberg, and \\ S. Mark Spearing, Member, ASME
}

\begin{abstract}
Micron-scale characterization of mechanical stresses is essential for the successful design and operation of many micromachined devices. Here we report the use of Raman spectroscopy to measure the bending stresses in deep reactive-ion etched silicon flexures with a stress resolution of $\sim 10 \mathrm{MPa}$ and spatial resolution of $\sim 1 \mu \mathrm{m}$. The accuracy of the technique, as assessed by comparison to analytical and finite-element models of the deformation, is conservatively estimated to be $25 \mathrm{MPa}$. Implications for the use of this technique in microsystems design are discussed.

[995]
\end{abstract}

Index Terms-Microelectromechanical systems (MEMS), microRaman, reliability, stress.

\section{INTRODUCTION}

$\mathbf{M}$ ICROELECTROMECHANICAL systems (MEMS) can be subjected to mechanical loads during fabrication [1], deployment [2] and operation [3] and are susceptible to failure due to fracture [3], buckling [4], and stiction [5]. Assessment of the reliability of micromachined structures requires accurate characterization of mechanical stresses. Direct measurement of local stresses must often accompany analytical or numerical models for deformation due to imprecise knowledge of materials properties, inability to predict the magnitudes of intrinsic residual stresses, and to assist in microsystems design [6].

Several techniques now exist for measuring stresses in microscale structures. The ones commonly used in MEMS design include wafer curvature measurement, fabrication of passive strain sensors, bulge tests, bending tests, and resonant tests [7]. These techniques are primarily used to characterize residual stresses in thin-film structures with a lateral spatial resolution typically exceeding tens of microns.

The increasing range of applications for MEMS, especially in power generation and propulsion [8], has led to growing interest in structures with critical dimensions in the range 0.1 to $10 \mathrm{~mm}$.

Manuscript January 16, 2003; revised May 16, 2003. The structures were fabricated at the Microsystems Technology Laboratories at the Massachusetts Institute of Technology (MIT), as part of a previous study, with financial support and encouragement from Professor S. Senturia. The micro-Raman spectroscopy was supported by the National Science Foundation NIRT ECS-0210752. Subject Editor W. N. Sharpe, Jr.

V. T. Srikar was with the Department of Aeronautics and Astronautics, Massachusetts Institute of Technology, Cambridge, MA 02139 USA. He is now with the Department of Mechanical Engineering, McGill University, Montreal, Canada (e-mail: srikar.vengallatore@mcgill.ca).

A. K. Swan and M. S. Ünlü are with the Department of Electrical and Computer Engineering, Boston University, Boston, MA 02215 USA.

B. B. Goldberg is with the Department of Physics and Department of Electrical and Computer Engineering, Boston University, Boston, MA 02215 USA.

S. M. Spearing is with the Department of Aeronautics and Astronautics, Massachusetts Institute of Technology, Cambridge, MA 02139 USA.

Digital Object Identifier 10.1109/JMEMS.2003.820280

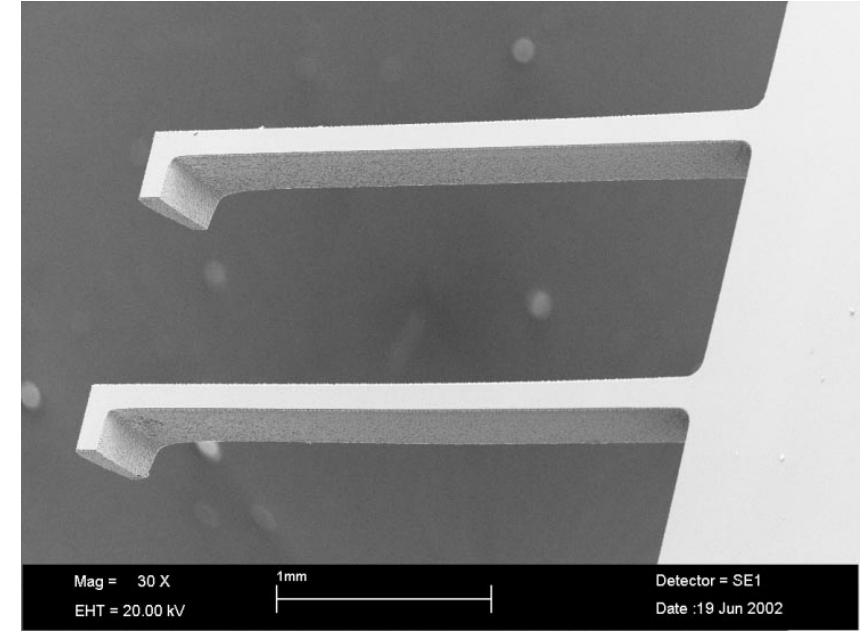

Fig. 1. Scanning electron micrograph of deep reactive-ion etched singlecrystal silicon flexures. The scale bar represents $1 \mathrm{~mm}$.

Consider, for example, the fluidic package of recently developed silicon microrocket and microengine devices that consists of metal tubes joined to silicon structures using glass seals. Numerical analysis and observations of brittle fracture indicate large stresses, with significant gradients over micron distances, in this composite structure [9]. The existing techniques cannot readily be used to measure such stresses.

There is a clear need for new experimental techniques capable of characterizing residual and applied stresses with micron spatial resolution in micromachined structures. A possible candidate is micro-Raman spectroscopy. It is well known that spectroscopy can be used to characterize stresses with micron spatial resolution in macroscale structures and silicon microelectronic devices [10]-[28]. The few existing spectroscopic characterization studies of MEMS [29]-[33] do not comprehensively evaluate the utility and limitations of this technique in microsystems design. In this paper, we present an evaluation of micro-Raman stress measurements by characterizing the bending stresses in micromachined single-crystal silicon flexures.

\section{FABRicAtion OF Silicon FleXURES}

The flexures are monolithic single-crystal silicon structures fabricated using a single-mask deep reactive-ion etching process (see Fig. 1). Each flexure is $3000 \mu \mathrm{m}$ long, $480 \mu \mathrm{m}$ wide, and the radius of curvature of the fillet at the root is $65 \mu \mathrm{m}$. The cross section of the flexures is not perfectly rectangular: the top surface is $150 \mu \mathrm{m}$ thick, while the thickness of the bottom surface varies between 120 and $130 \mu \mathrm{m}$. All the measurements and 


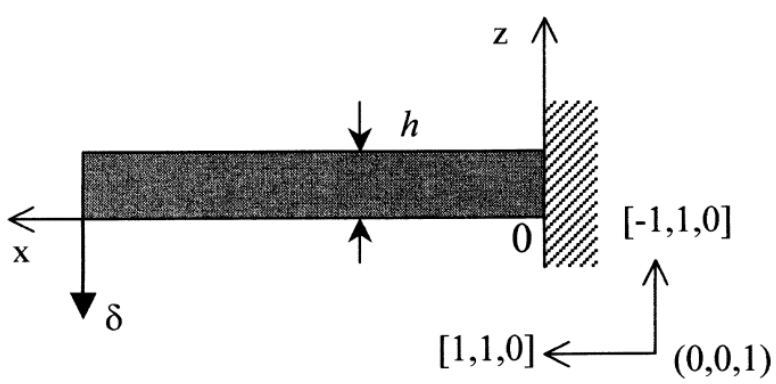

Fig. 2. Model of the flexure as a cantilevered beam of thickness $h$ and length $L$, subjected to a tip displacement $\delta$. The crystallography of the specimen is indicated.

calculations reported in this paper refer to stresses in the vicinity of the top surface.

The starting material for fabrication was a double-polished, $(1,0,0)$-oriented, single-crystal silicon wafer. One surface was coated with photoresist AZ4620 spun to a speed of $4000 \mathrm{rpm}$ to achieve a final thickness of $\sim 10 \mu \mathrm{m}$. After photolithography and development, the patterned photoresist served as a masking material during the etching of the silicon substrate. Deep-reactive-ion etching was performed in a Surface Technology Systems inductively coupled plasma reactor, with alternating cycles of reactive-ion etching in $\mathrm{SF}_{6}$ plasma and polymer deposition from a $\mathrm{CF}_{4}$ plasma. The etch rate was $\sim 2.2 \mu \mathrm{m} / \mathrm{min}$ and the selectivity of silicon to photoresist approximately 70:1 [34]. The unetched photoresist was subsequently stripped in oxygen plasma.

After fabrication, a $1 \mathrm{~mm}$ diameter silicon sphere was inserted between the tips of two adjacent flexures. The deflections of the tip $(\delta)$ of the flexures were measured to be $69.5 \mu \mathrm{m}$ and $87 \mu \mathrm{m}$, respectively. The error in the measurement of $\delta$ is estimated to be $\sim 1 \mu \mathrm{m}( \pm 1.5 \%)$. Care was taken to avoid torsional loads during the loading process.

\section{MODELS FOR MECHANICAL DEFORMATION}

\section{A. Euler-Bernoulli Beam}

As a first approximation, we model each flexure as an EulerBernoulli beam of uniform rectangular cross-section of length $L$, width $b$, thickness $h$, and tip deflection $\delta$, as shown in Fig. 2. This implies the following set of assumptions [35]: 1) plane sections remain plane; 2) the beam is initially straight; 3 ) the beam is homogeneous and perfectly elastic; 4) the flexure deforms in pure bending; and 5) Poisson contraction (or expansion) is not constrained. This last assumption is valid for narrow beams $(b<5 h)$ and because the Poisson ratio of silicon for the orientation under consideration is only 0.064 [36]. The choice of single-crystal silicon as the starting material ensures that the flexures are not subjected to any residual stresses.

The theory of small deformations is valid since $L>20 h$ and $L>40 \delta$. When the flexure is deformed in pure bending, we expect a uniaxial stress $\left(\sigma_{x x}\right)$ that is tensile at the surface $z=h$, compressive at the surface $z=0$, and zero at $z=(h / 2)$. All other stress components are identically zero. The spatial variation of $\sigma_{x x}$ is then given by [35]

$$
\sigma_{x x}(x, z)=\frac{3 E \delta h}{2 L^{3}}(L-x)\left[-1+\frac{2 z}{h}\right] .
$$

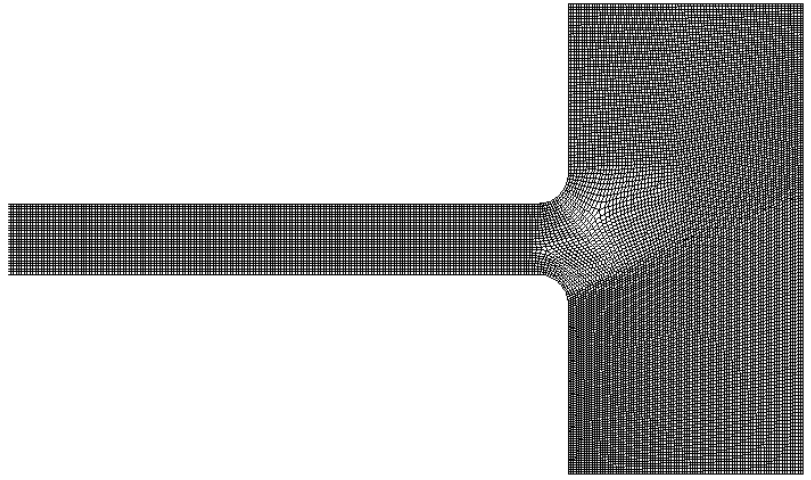

Fig. 3. A portion of the finite element mesh used to analyze the deformation of the flexure. Clamped boundary conditions were imposed on the right end of the mesh.

The value of the Young's modulus $(E)$ along the $[1,1,0]$-direction in the $(0,0,1)$-plane of silicon is used in (1) to match the crystallography of the structure under consideration. This value has been estimated to be $168.9 \mathrm{GPa}$ [36].

Equation (1) is invalid in a region of order $h$ in the vicinity of the root as a consequence of Saint-Venant's principle [35]. The assumptions of plane sections remaining plane, and of uniaxial stress distribution, are violated in this region due to the finite compliance of the supporting silicon structure and any stress concentrations at the fillet. The stress components $\sigma_{z z}$ and $\sigma_{x z}$ assume nonzero values at the root. In order to take these effects into consideration, numerical analysis was performed using the method of finite elements

\section{B. Finite-Element Analysis}

The deformation of the flexure was analyzed using a general-purpose finite-element solver (ABAQUS [37]). The cross section of the flexure was assumed to be rectangular and a twodimensional (2-D) model was created using four-node plane stress quadrilateral elements. A portion of the supporting silicon structure was also meshed to account for any support compliance (see Fig. 3). Clamped boundary conditions were imposed on the end of the support. Silicon was modeled as an isotropic solid ( $E=169 \mathrm{GPa}$ and $\nu=0.07$ ) and a total of 26520 elements were employed in the mesh.

The tip of the beam was subjected to static displacements of $69.5 \mu \mathrm{m}$ and $87 \mu \mathrm{m}$ by applying appropriate point forces. The quality of the mesh was verified by first analyzing the stresses without any support compliance. The normal stress, $\sigma_{x x}$, matched (1) to $\sim 3 \%$. With the same mesh density, but with the addition of the support structure, the values of $\sigma_{x x}$ matched (1) to $\sim 7 \%$, indicating only a modest support compliance in this structure. The stresses $\sigma_{z z}$ and $\sigma_{x z}$ were found to be localized at the root and assume nonzero values only on the domain $0<x<100 \mu \mathrm{m}$.

\section{The Strain Tensor}

The analysis of the previous sections refers to a coordinate system with unit vectors parallel to $[1,1,0],[-1,1,0]$ and $[0,0,1]$, such that $\sigma_{x x}$ is parallel to $[1,1,0]$. The use of the crystal coordinates, with unit vectors parallel to $[1,0,0]$, $[0,1,0]$, and $[0,0,1]$, is more convenient in spectroscopic 
TABLE I

ELASTIC CONSTANTS OF SiLICON [36]

\begin{tabular}{c|c}
\hline Property & Value \\
\hline $\mathrm{S}_{11}$ & $7.68 \mathrm{TPa}^{-1}$ \\
\hline $\mathrm{S}_{12}$ & $-2.14 \mathrm{TPa}^{-1}$ \\
\hline $\mathrm{S}_{44}$ & $12.6 \mathrm{TPa}^{-1}$ \\
\hline
\end{tabular}

analysis. A simple coordinate transformation results in the following stress $\left(\sigma_{i j}\right)$ and strain $\left(\epsilon_{i j}\right)$ tensors in the crystal coordinate system [38]

$$
\begin{aligned}
\sigma_{i j} & =\left[\begin{array}{ccc}
0.5 \sigma_{x x} & 0.5 \sigma_{x x} & 0 \\
0.5 \sigma_{x x} & 0.5 \sigma_{x x} & 0 \\
0 & 0 & 0
\end{array}\right] \\
\varepsilon_{i j} & =\left[\begin{array}{ccc}
\frac{\left(S_{11}+S_{12}\right)}{2} \sigma_{x x} & \frac{S_{44}}{2} \sigma_{x x} & 0 \\
\frac{S_{44}}{2} \sigma_{x x} & \frac{\left(S_{11}+S_{12}\right)}{2} \sigma_{x x} & 0 \\
0 & 0 & S_{12} \sigma_{x x}
\end{array}\right]
\end{aligned}
$$

where $S_{11}, S_{12}$, and $S_{44}$ are elements of the compliance tensor of silicon (see Table I).

\section{RAMAN SPECTROSCOPY: THEORY}

The phenomenon of Raman scattering, and the effects of mechanical stress on the Raman spectrum of single-crystal silicon, have been extensively studied and documented [10]-[26]. The principal results pertinent to the analysis of the flexure are summarized below.

Monochromatic radiation that is incident on the surface of a solid can, in general, undergo transmission, absorption, and scattering. In Stokes-Raman scattering the incident photons couple with phonon-induced changes in the polarizability of the solid, causing the scattered photons to loose a quantum of lattice energy or phonon relative to the incident photons. Conservation of momentum dictates that only phonons close to the $\Gamma$-point of the Brillouin zone are excited in such processes. Similarly, conservation of energy implies that the frequency $(\omega)$ of the excited phonons is given by [10]

$$
\omega=\left[\frac{1}{\lambda_{i}}-\frac{1}{\lambda_{s}}\right]
$$

where $\lambda_{i}$ and $\lambda_{s}$ are the wavelength of the incident and scattered radiation, respectively. The quantity $\omega$ is called the Raman shift and is typically reported in units of $\mathrm{cm}^{-1}$. The Raman spectrum, then, is a graph of the scattered intensity as a function of the Raman shift and contains information about the physical and chemical characteristics of the solid.

A schematic illustration of spectroscopy in the backscattering geometry is shown in Fig. 4. Stress-free single-crystal silicon has three degenerate Raman-active optical phonon modes: two transverse and one longitudinal. When the incident and scattered

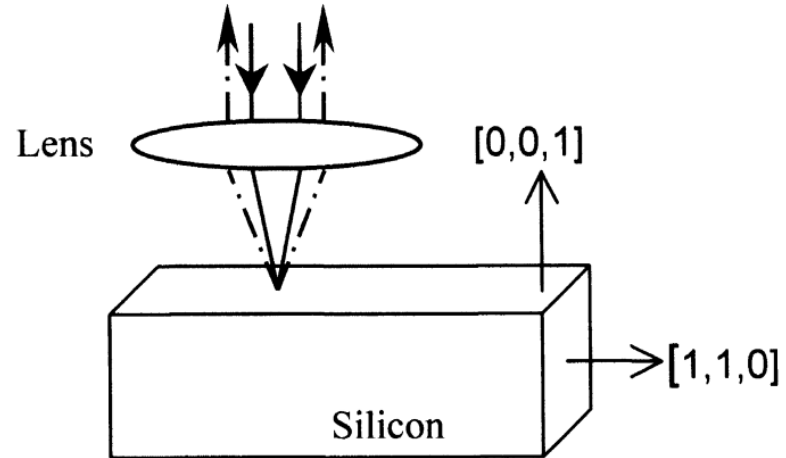

— Incident radiation

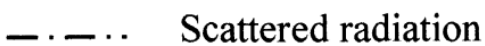

Fig. 4. Schematic illustration of backscattering raman spectroscopy. Each spectrum corresponds to a stress measurement from a volume $\sim 1 \mu \mathrm{m}^{3}$ of silicon.

radiation are both parallel to $[0,0,1]$, the transverse modes are polarized along $[1,0,0]$ and $[0,1,0]$, respectively, and the longitudinal mode is polarized along $[0,0,1]$. The scattering efficiency, $I$, can be expressed as [19]

$$
I=A \sum_{j}\left|e_{i}^{t} \cdot \mathbf{R}_{j} \cdot \mathbf{e}_{s}\right|^{2} \quad j=1,2,3 .
$$

Here $A$ is a constant, $\mathbf{e}_{i}$ and $\mathbf{e}_{s}$ are the polarization vectors of the incident and scattered light, respectively, the superscript $t$ denotes the transposed vector, and $\mathbf{R}_{j}(j=1,2,3)$ are the Raman polarizability tensors. These second-rank tensors are given by [11]

$$
\begin{aligned}
\mathbf{R}_{1} & =\left(\begin{array}{lll}
0 & 0 & 0 \\
0 & 0 & 1 \\
0 & 1 & 0
\end{array}\right) \\
\mathbf{R}_{2} & =\left(\begin{array}{lll}
0 & 0 & 1 \\
0 & 0 & 0 \\
1 & 0 & 0
\end{array}\right) \\
\mathbf{R}_{3} & =\left(\begin{array}{lll}
0 & 1 & 0 \\
1 & 0 & 0 \\
0 & 0 & 0
\end{array}\right)
\end{aligned}
$$

corresponding to the crystallographic directions $[1,0,0]$, $[0,1,0]$, and $[0,0,1]$, respectively. For backscattering from the $(0,0,1)$-plane (i.e., both $\mathbf{e}_{i}$ and $\mathbf{e}_{s}$ have the form $[\alpha, \beta, 0]$, with arbitrary $\alpha$ and $\beta$ ), (4) and (5) together dictate that only the longitudinal mode has a nonzero scattering efficiency. The Raman spectrum consists of a single peak with a Raman shift $\left(\omega_{0}\right)$ of $\sim 521 \mathrm{~cm}^{-1}$.

In general, the degeneracy of the Raman frequencies is lifted, and the Raman shifts acquire new values $\left(\omega_{j}\right)$ for each of the three modes, when silicon is subjected to external mechanical stresses. The change in the Raman shifts $(\Delta \omega)$ is given by [18]

$$
\Delta \omega_{j}=\omega_{j}-\omega_{0} \cong \frac{\lambda_{j}}{2 \omega_{0}} \quad j=1,2,3
$$


TABLE II

PHONON DEFORMATION POTENTIALS (PDPS) OF SILICON

\begin{tabular}{c|c|c}
\hline Property & $\begin{array}{c}\text { PDPs of } \\
\text { Chandrasekhar } \text { et al. } \text { [15] }\end{array}$ & $\begin{array}{c}\text { PDPs of } \\
\text { Anastassakis } \text { et al. [16] }\end{array}$ \\
\hline$p / \omega_{0}^{2}$ & $-1.49 \pm 0.06$ & $-1.85 \pm 0.06$ \\
\hline$q / \omega_{0}^{2}$ & $-1.97 \pm 0.06$ & $-2.31 \pm 0.06$ \\
\hline$r / \omega_{0}^{2}$ & $-1.61 \pm 0.06$ & $-0.71 \pm 0.02$ \\
\hline
\end{tabular}

where $\lambda_{j}(j=1,2,3)$ are the eigenvalues of the matrix shown in (7) at the bottom of the page. Here, $p, q$, and $r$ are the phonon deformation potentials (PDPs) of silicon (see Table II) and $\epsilon_{i j}$ are elements of the strain tensor referred to the crystal coordinate system. For the strain tensor given by (2), the eigenvalues of (7) can be readily shown to be

$$
\begin{aligned}
\lambda_{1} & =\frac{1}{2}\left[p \sigma_{x x}\left(S_{11}+S_{12}\right)+q \sigma_{x x}\left(S_{11}+3 S_{12}\right)+r \sigma_{x x} S_{44}\right] \\
\lambda_{2} & =\frac{1}{2}\left[p \sigma_{x x}\left(S_{11}+S_{12}\right)+q \sigma_{x x}\left(S_{11}+3 S_{12}\right)-r \sigma_{x x} S_{44}\right] \\
\lambda_{3} & =\left[q\left(S_{11}+S_{12}\right)+p S_{12}\right] \sigma_{x x}
\end{aligned}
$$

corresponding to the eigenvectors $[1,1,0],[-1,1,0]$ and $[0,0,1]$ respectively. The applied stress can also lead to a change in the Raman polarizability tensors. This change is assumed to be a linear combination of the stress-free tensors given by [25]

$$
\mathbf{R}_{k}^{\sigma}=\left(n_{1}\right)_{k} \mathbf{R}_{1}+\left(n_{2}\right)_{k} \mathbf{R}_{2}+\left(n_{3}\right)_{k} \mathbf{R}_{3} \quad k=1,2,3
$$

where the superscript $\sigma$ denotes the stressed-value of the tensor; $\left(n_{1}\right)_{k},\left(n_{2}\right)_{k}$, and $\left(n_{3}\right)_{k}$ are the directional cosines between the kth eigenvector of (8) and the three stress-free eigenvectors. From (5), (8) and (9), we obtain

$$
\begin{aligned}
\mathbf{R}_{1}^{\sigma} & =\frac{1}{\sqrt{2}}\left(\begin{array}{lll}
0 & 0 & 1 \\
0 & 0 & 1 \\
1 & 1 & 0
\end{array}\right) \\
\mathbf{R}_{2}^{\sigma} & =\frac{1}{\sqrt{2}}\left(\begin{array}{ccc}
0 & 0 & 1 \\
0 & 0 & -1 \\
1 & -1 & 0
\end{array}\right) \\
\mathbf{R}_{3}^{\sigma} & =\left(\begin{array}{lll}
0 & 1 & 0 \\
1 & 0 & 0 \\
0 & 0 & 0
\end{array}\right) .
\end{aligned}
$$

As before, for arbitrary $\mathbf{e}_{i}$ and $\mathbf{e}_{s}$ propagating along $[0,0,1]$, the Raman spectrum contains a single peak corresponding to the eigenvector $[0,0,1]$. From (8), therefore, the change in the Raman shift is simply

$$
\Delta \omega=\frac{\lambda_{3}}{2 \omega_{0}}=\frac{q\left(S_{11}+S_{12}\right)+p S_{12}}{2 \omega_{0}} \sigma_{x x} .
$$

For $\omega_{0}=521 \mathrm{~cm}^{-1}$, and using the phonon deformation potentials of Anastassakis et al. [16], we obtain

$$
\sigma_{x x} \cong-434 \Delta \omega
$$

and using the phonon deformation potentials of Chandrasekhar et al. [15], we obtain

$$
\sigma_{x x} \cong-518 \Delta \omega .
$$

In both (12) and (13), $\sigma_{x x}$ is expressed in units of megapascals and $\Delta \omega$ in $\mathrm{cm}^{-1}$.

Corresponding to any measured change in the Raman shift, therefore, is a uniquely determined value of the local uniaxial stress in the flexure. The locality of the measurement is determined by the spot size, which is $1.3 \mu \mathrm{m}$ in our measurements.

\section{RAMAN SPECTROSCOPY: MEASUREMENTS}

Raman spectroscopy was performed using a commercial Renishaw spectrometer (model $1000 \mathrm{~B}$ ) in the backscattering configuration. The spectral excitation was produced by utilizing the $514 \mathrm{~nm}$ line of an argon-ion laser. The incident light, polarized parallel to the $[-1,1,0]$-direction, was focused on the $(0,0,1)$-surface of the structure using a $50 \mathrm{X}$ microscope objective, and the scattered light was collected using the same objective, as illustrated in Fig. 4. The direction of polarization of the scattered light was not analyzed. The spot size of the incident beam is estimated to be $1.3 \mu \mathrm{m}$, and the sampling depth is $\sim 0.8 \mu \mathrm{m}$, based on the penetration distance of $514 \mathrm{~nm}$ light in silicon [10]. Each spectrum therefore represents a stress measurement from a volume $\sim 1 \mu \mathrm{m}^{3}$ of silicon.

All the measurements were performed at room temperature (300 K). The incident power was chosen to be $2 \mathrm{~mW}$, corresponding to a power density of $300 \mathrm{~kW} / \mathrm{cm}^{2}$, to minimize local heating due to the laser probe. The time for collecting a single spectrum was between 2 and 4 s. Fig. 5 shows the Raman spectrum of stress-free single-crystal silicon. The peak frequency $\left(\omega_{0}\right)$ was determined to be $520.91 \pm 0.01 \mathrm{~cm}^{-1}$ by fitting the intensity to a Lorentzian function [39]. The coefficient of determination of the fit was 0.99 and the full width at half maximum intensity was $\sim 3.5 \mathrm{~cm}^{-1}$. Fig. 6 shows the Raman shifts obtained from a line scan on stress-free silicon. The spectral resolution is estimated to be $\pm 0.02 \mathrm{~cm}^{-1}$ from this measurement. This corresponds to a stress resolution of $\sim 10 \mathrm{MPa}$ from (12) and (13).

The bending stresses were characterized by performing line scans across the thickness at several locations along the axis

$$
\varphi_{i j}=\left[\begin{array}{ccc}
p \varepsilon_{11}+q\left(\varepsilon_{22}+\varepsilon_{33}\right) & 2 r \varepsilon_{12} & 2 r \varepsilon_{13} \\
2 r \varepsilon_{12} & p \varepsilon_{22}+q\left(\varepsilon_{33}+\varepsilon_{11}\right) & 2 r \varepsilon_{23} \\
2 r \varepsilon_{13} & 2 r \varepsilon_{23} & p \varepsilon_{33}+q\left(\varepsilon_{11}+\varepsilon_{22}\right)
\end{array}\right]
$$




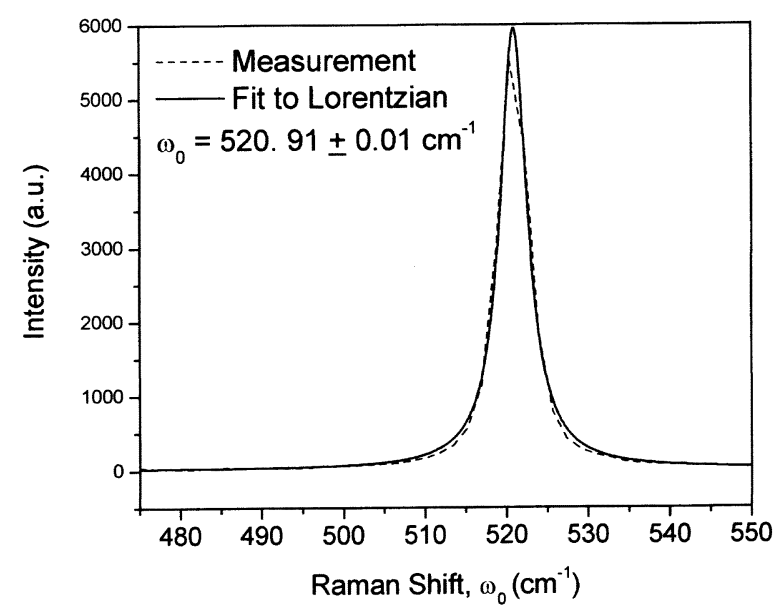

Fig. 5. The raman spectrum of stress-free single-crystal silicon.

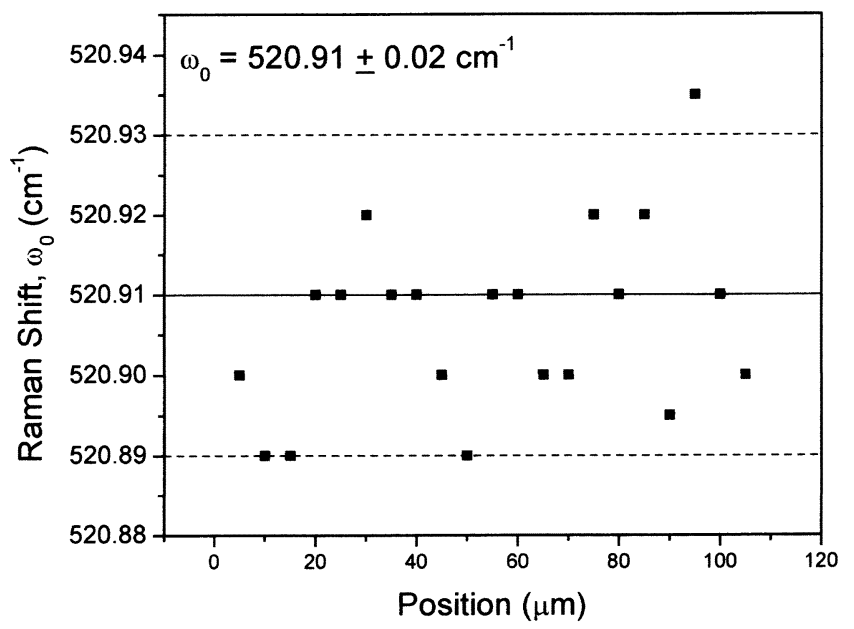

Fig. 6. Raman shifts determined at several locations on a stress-free silicon wafer from a line scan. The spectral resolution of $0.02 \mathrm{~cm}^{-1}$ corresponds to a stress resolution of $\sim 10 \mathrm{MPa}$.

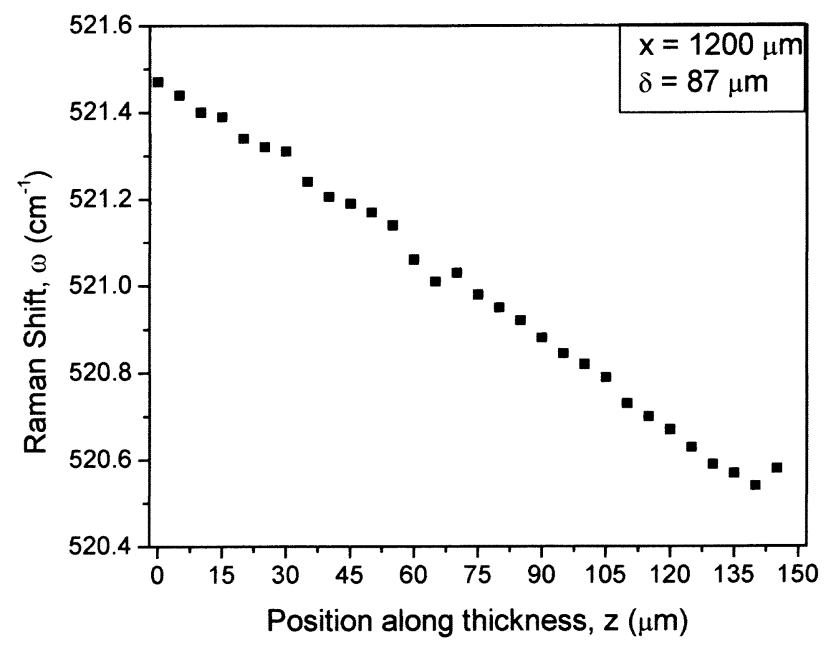

Fig. 7. Raman shift measured as a function of position along thickness using a line scan. (For this scan, $\omega_{0}=521.01 \mathrm{~cm}^{-1}$ ).

of the flexures. Each scan commenced at the compressively stressed edge. A representative graph of the measured Raman shifts is shown in Fig. 7. The changes in the Raman shifts (with reference to the measured stress-free value) lie in the range

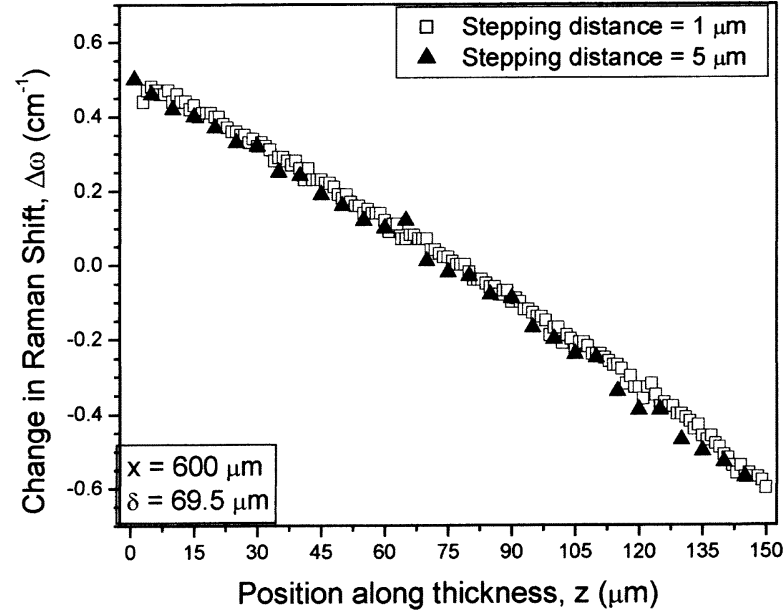

Fig. 8. Line scans of the same location, using different stepping distances, demonstrate that the measurements are repeatable to $\pm 0.02 \mathrm{~cm}^{-1}$.

$-0.6 \mathrm{~cm}^{-1}$ to $+0.6 \mathrm{~cm}^{-1}$. Two independent line scans of the same location, with stepping distances of $1 \mu \mathrm{m}$ and $5 \mu \mathrm{m}$, agree to $\pm 0.02 \mathrm{~cm}^{-1}$ (see Fig. 8), confirming the repeatability of our measurements.

The measured changes in the Raman shift were converted into stress values using (12) and (13). The stress profiles at distances of $600 \mu \mathrm{m}$ and $1200 \mu \mathrm{m}$ are shown in Figs. 9 and 10, respectively. The predictions of the Euler-Bernoulli model and finite-element models are also shown. The stress values obtained using the phonon deformation potentials of Anastassakis et al. [16] provide a consistently better match to model predictions.

\section{DISCUSSION}

\section{A. Resolution and Accuracy}

Fig. 6 indicates that the spectral resolution of the measurement is $0.02 \mathrm{~cm}^{-1}$. From (12) and (13), this corresponds to a stress resolution of $\sim 10 \mathrm{MPa}$ and represents the smallest relative stress difference that can be measured in our experiments. The determination of absolute stress value depends also on the knowledge of the elastic constants and phonon deformation potentials of silicon.

The elastic constants of silicon have been measured to $\pm 0.25 \%$ [40]; multiple, independent measurements confirm the values of Table I to be accurate. On the other hand, the phonon deformation potentials are known only to $\pm 3 \%$ and two independent measurements report values that differ, on average, by about $20 \%$ (see Table II). Chandrasekhar, Renucci, and Cardona [15] measured one set using backscattering experiments, while Anastassakis, Cantarero and Cardona [16] revised these values using transmission spectroscopy. The latter values are expected to be more accurate since they exclude any possible surface relaxation effects. Figs. 9 and 10 confirm this expectation.

Ideally, the accuracy of our measurements should be assessed by comparison to results from an alternate, well-established, technique. Lacking any convenient such alternative, we assess the accuracy by comparing the measurements to the predictions of finite element analysis. Using the phonon deformation potentials of Anastassakis et al. [16], the spectroscopic measurements 


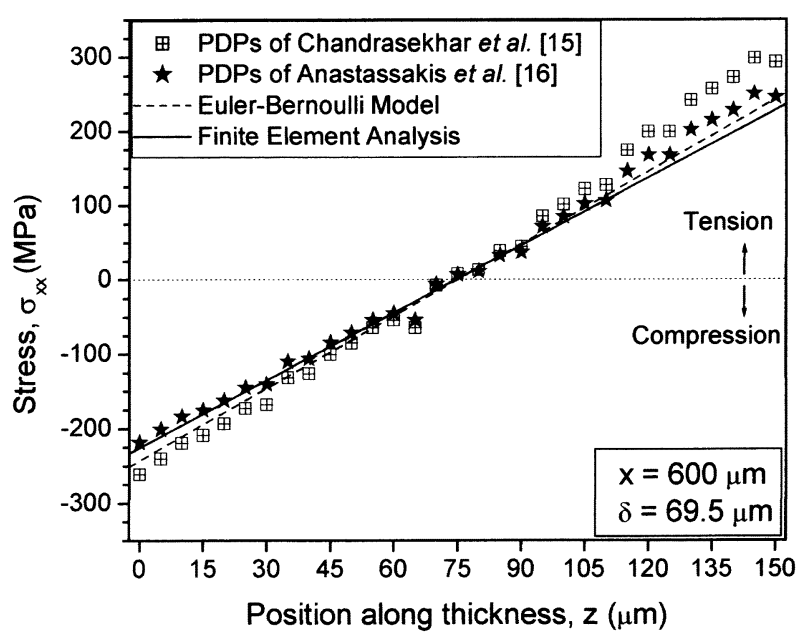

Fig. 9. Stress profile across the thickness of the beam at $x=600 \mu \mathrm{m}$. The phonon deformation potentials (PDPs) of Anastassakis et al. [16] provide a closer match to the predictions of the finite-element analysis.

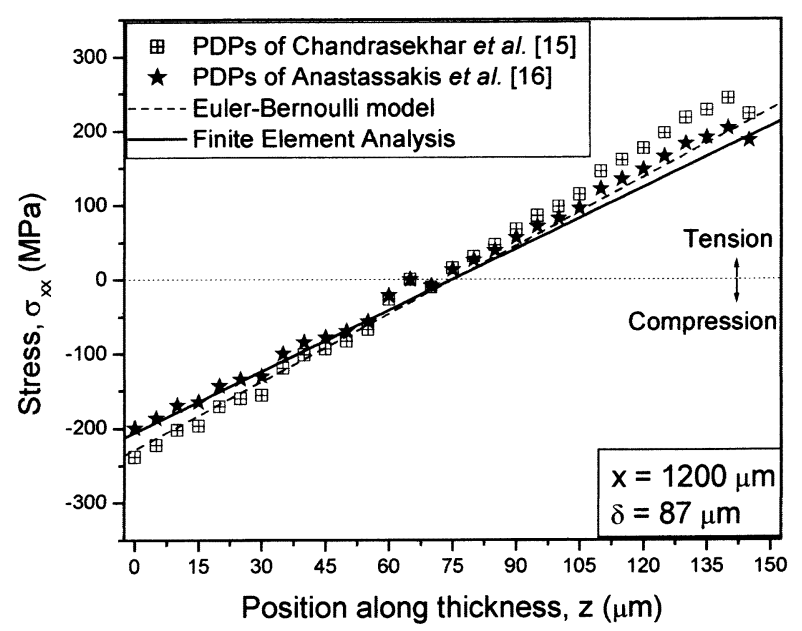

Fig. 10. Stress profile across the thickness of the beam at $x=1200 \mu \mathrm{m}$. Again, the phonon deformation potentials (PDPs) of Anastassakis et al. [16] provide a closer match to the predictions of the finite-element analysis.

match the analysis to $10 \mathrm{MPa}$ in compression but overestimate by $\sim 25 \mathrm{MPa}$ in tension. The reason for this mild asymmetry is unclear at present. We have considered, and discounted, the following possibilities.

First, the Raman shift is known to be sensitive to ambient conditions. The reference value, $\omega_{0}$, was measured prior to every scan to account for such effects. There was no systematic drift during the line scans as demonstrated by Fig. 6. Second, we consider errors due to specimen orientation such that a scan along the $z$-axis was also a scan along the $x$-axis. An error of $15 \mathrm{MPa}$ in stress measurement requires a misalignment greater than $30^{\circ}$. This is unreasonable since we estimate any such alignment errors to be less than $2^{\circ}$ in our setup. Third, the scan direction (i.e., the $z$-axis of Fig. 2) is not exactly perpendicular to the neutral axis due to the deformation of the structure. It is simple to show that this geometric effect results in a correction less than $1 \mathrm{MPa}$, and can be neglected.

Fourth, the cross-section of the flexure is trapezoidal, and not rectangular, as mentioned earlier. Microscopic examinations of nominally identical flexures tested to fracture in bending experiments indicated that the cross-section is still symmetric about the plane $z=h / 2$ [34]. Further, the position of zero stress in Figs. 9 and 10 coincides with the nominal position of the neutral axis $(z=75 \mu \mathrm{m})$. We conclude that the shape is not solely responsible for the observed asymmetry.

Fifth, it is possible that the flexure suffered unintentional torsional loads, or out-of-plane bending, during deformation. Again, the coincidence of the zero-stress locations with $z=$ $h / 2$ is an argument against any such additional deformation. It must be noted that, for the crystallography and scattering geometry under consideration, some stress states (such as pure shear) will induce no change in the Raman shift of the longitudinal mode, and cannot be ruled out based purely on the position of the neutral axis. For the same reason, however, such stresses cannot account for the observed asymmetry in tension and compression.

Finally, the structures were prepared using deep reactive-ion etching and it is possible that processing-induced residual stresses are present in the flexures. Any such stresses are expected to occur at the beam edges and should affect the tensile and compressive regions to the same extent. No significant changes of the Raman shift were detected during measurements of undeformed beams. Residual stresses can therefore be excluded from consideration.

We conclude for the present that the accuracy of our measurement lies in the range $10 \mathrm{MPa}$ to $25 \mathrm{MPa}$. The expectation is that future measurements will result in narrower bounds. It is worth emphasizing that even the conservative estimate of $25 \mathrm{MPa}$ is comparable to the accuracy achieved using other methods of microscale stress measurement and is sufficient for applications in microsystem design [7].

\section{B. Complex Stress States}

The interpretation of stress fields using spectroscopic data requires careful identification of the form of the stress tensor. This is generally true of other microscale stress measurement techniques [7]. Significant errors can occur when such identification is incorrectly made.

As an illustrative example, consider the stresses at the root of the flexure. The finite element analysis indicates normal $\left(\sigma_{x x}\right.$ and $\left.\sigma_{z z}\right)$ and shear $\left(\sigma_{x z}\right)$ components with magnitudes exceeding $100 \mathrm{MPa}$. If, instead, the stress field is assumed to be uniaxial and the spectroscopic data converted using (12), the resultant errors can exceed $100 \mathrm{MPa}$ as shown in Fig. 11.

Even when correctly identified, it is nontrivial to extract multiple stress components from backscattering measurements of Raman shifts. One possibility is to perform self-consistent numerical and spectroscopic analysis to deduce the stress tensor [17]. The other is to perform additional spectroscopic measurements involving the polarizations of, and the relative angles between, the incident and scattered radiation. Such studies are topics of current research [25], [26], [41]. The use of time-resolved spectroscopy to measure dynamic stresses in rotating micromachinery is also being explored.

Many materials including zirconia [21], silicon carbide [22], aluminum nitride [23], and diamond [24] are Raman active 


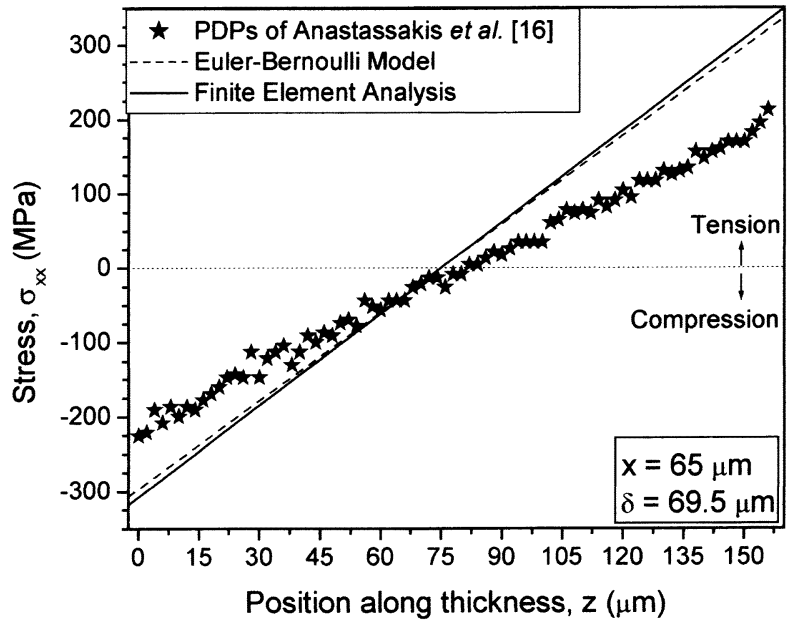

Fig. 11. Stress profile at the root of the beam assuming a uniaxial stress state. The inability to resolve complex stresses represents a significant limitation of this technique.

and can be characterized using spectroscopy. Extensions to polycrystalline materials have also been attempted, although confinement effects due to the finite size of the grains can lead to asymmetric peak broadening and complicate the extraction of stress information from such measurements [27], [28], [31]. Many common metals are typically not Raman active and cannot directly be characterized using spectroscopy.

\section{IMPLICATIONS FOR USE IN MEMS DESIGN}

The preceding sections demonstrate that Raman spectroscopy can be used to characterize simple stress states in micromachined single-crystal silicon structures with micrometer spatial resolution. Previously measured values of elastic constants and phonon deformation potentials can be used to obtain a stress sensitivity of $\sim 10 \mathrm{MPa}$, which is usually adequate for designing bulk-micromachined devices. The measurements are rapid, nondestructive, and are made using commercially available instruments.

Whereas this combination of attributes is unique among microscale stress measurement techniques, the inability to resolve complex stresses significantly limits the utility of micro-Raman spectroscopy in design. Fig. 11 highlights this observation. The maximum bending stresses, and the sites for nucleation of brittle fracture [34], occur at the root of the silicon flexures. Yet, backscattering micro-Raman measurements are incapable of accurately characterizing these critical stresses.

Until further advances permit tensorial stress analysis, it is expected that spectroscopy will be most effective when used in conjunction with numerical analysis. This is especially true if one parameter, such as the coefficient of thermal expansion $(\alpha)$ of one material in a composite structure, is unknown. One example of current interest is the design of glass-silicon hermetic seals in the silicon microrocket device mentioned earlier [9]. The sealing process involves a thermal excursion of over $1000{ }^{\circ} \mathrm{C}$; the resultant thermal stresses cannot be accurately calculated since $\alpha_{\text {glass }}$ is not well characterized. Hence, we are employing parametric finite-element computations to predict the (uniaxial) stresses in silicon using systematically varied values of $\alpha_{\text {glass }}$. The value that leads to the closest match with spectroscopically measured stresses will be used in further design [42].

The use of spectroscopy to determine optimal processing parameters, such as annealing conditions to minimize intrinsic residual stresses, defines a second category of applications. Because the phonon deformation potentials are sensitive to structural details (grain size; type and concentration of dopants), the values listed in Table II cannot be used. Changes in the Raman shift will have to be calibrated as functions of the applied stress for each specific set of structures of interest [43].

Finally, it is instructive to note that recent progress in $\mathrm{x}$-ray optics has led to the development of another stress measurement technique - scanning X-ray microdiffraction $(\mu \mathrm{SXRD})$ - that can determine the entire stress tensor, with micrometer spatial resolution, in mono- and polycrystalline specimens [44]. However, the use of $\mu \mathrm{SXRD}$ in microsystem design is limited by access to high quality synchrotron sources.

\section{CONCLUSION}

Micron-scale characterization of mechanical stresses is essential for the reliable operation of many micromachines. The uniaxial bending stresses in a micromachined single-crystal silicon were characterized using Raman spectroscopy. The stress resolution $(\sim 10 \mathrm{MPa})$ of this technique is comparable, and the spatial resolution $(\sim 1 \mu \mathrm{m})$ considerably superior, to those of the other commonly used microscale stress measurement methods. The accuracy of the technique, as assessed by comparison to analytical and numerical analysis of the deformation, is conservatively estimated to be $25 \mathrm{MPa}$. The utility of this technique in microsystem design was discussed.

\section{ACKNOWLEDGMENT}

The authors are indebted to Ms. A. Mracek of MIT for performing the scanning electron microscopy.

\section{REFERENCES}

[1] M. J. Madou, Fundamentals of Microfabrication. Boca Raton, FL: CRC, 2002.

[2] V. T. Srikar and S. D. Senturia, "The reliability of microelectromechanical systems in shock environments," J. Microelectromech. Syst., vol. 11, pp. 206-214, 2002.

[3] K.-S. Chen, A. A. Ayon, and S. M. Spearing, "Controlling and testing the strength of silicon on the mesoscale," J. Amer. Ceram. Soc., vol. 83, pp. 1476-1484, 2000

[4] S. D. Senturia, Microsystems Design. Norwell: Kluwer, 2001.

[5] C. H. Mastrangelo, "Adhesion-related failure mechanisms in micromechanical devices," Tribol. Lett., vol. 3, pp. 223-238, 1997.

[6] V. T. Srikar and S. M. Spearing, "Materials selection in micromechanical design: An application of the ashby approach," J. Microelectromech. Syst., vol. 12, pp. 3-10, Feb. 2003.

[7] - "A critical review of microscale mechanical testing methods used in the design of MEMS," Exper. Mech., 2003.

[8] A. H. Epstein and S. D. Senturia, "Macro power from micro machines," Science, vol. 276, p. 1211, 1997

[9] Y. Peles, V. T. Srikar, T. S. Harrison, C. Protz, A. Mracek, and S. M. Spearing, "Fluidic packaging of microengine and microrocket devices for high-pressure and high-temperature operation," J. Microelectromech. Syst., vol. 11, 2002. 
[10] M. J. Pelletier, Analytical Applications of Raman Spectroscopy. Oxford, U.K.: Blackwell Science, 1999.

[11] R. Loudon, "The raman effect in crystals," Adv. Phys., vol. 13, pp. 423-482, 1964.

[12] E. Anastassakis, A. Pinczuk, E. Burstein, F. H. Pollack, and M. Cardona, "Effect of uniaxial stress on the raman spectrum of silicon," Solid State Commun., vol. 8, pp. 133-138, 1970.

[13] E. Anastassakis and E. Burstein, "Morphic effects I: Effects of external forces on photon-optical phonon interactions," J. Phys. Chem. Solids, vol. 32, pp. 313-326, 1971

[14] _ "Morphic effects II: Effects of external forces on the frequencies of the $q=0$ optical phonons," J. Phys. Chem. Solids, vol. 32, pp. 563-570, 1971.

[15] M. Chandrasekhar, J. B. Renucci, and M. Cardona, "Effects of interband excitations on raman phonons in heavily doped n-Si," Phys. Rev., vol. B17, pp. 1623-1633, 1978.

[16] E. Anastassakis, A. Cantarero, and M. Cardona, "Piezo-Raman measurements and anharmonic parameters in silicon and diamond," Phys. Rev., vol. B41, pp. 7529-7535, 1990.

[17] Q. Ma, S. Chiras, D. R. Clarke, and Z. Suo, "High resolution determination of the stress in individual interconnect lines and the variation due to electromigration," J. Appl. Phys., vol. 78, pp. 1614-1622, 1995.

[18] I. De Wolf, H. E. Maes, and S. K. Jones, "Stress measurements in silicon devices through raman spectroscopy: Bridging the gap between theory and experiment," J. Appl. Phys., vol. 79, pp. 7148-7156, 1996.

[19] E. Anastassakis, "Selection rules of raman scattering by optical phonons in strained cubic crystals," J. Appl. Phys., vol. 82, pp. 1582-1591, 1997.

[20] I. De Wolf, "Micro-Raman spectroscopy to study local mechanical stress in silicon integrated circuits," Semicond. Sci. Tech., vol. 11, pp. 139-154, 1996.

[21] J. G. Cai, Y. S. Raptis, and E. Anastassakis, "Stabilized cubic zirconia: A Raman study under uniaxial stress," Appl. Phys. Lett., vol. 62, pp. 2781-2783, 1993.

[22] S. Rohmfeld, M. Hundhausen, L. Ley, C. A. Zorman, and M. Mehregany, "Quantitative evaluation of biaxial strain in epitaxial 3C-SiC layers on Si(100) substrates by raman spectroscopy," J. Appl. Phys., vol. 91, pp. 1113-1117, 2002.

[23] A. Sarua, M. Kuball, and J. E. Van Nostrand, "Deformation potentials of the E2 (high) phonon mode of AIN," Appl. Phys. Lett., vol. 81, pp. 1426-1428, 2002.

[24] N. G. Ferreira, E. Abramof, N. F. Leite, E. J. Corat, and V. J. Trava-Airoldi, "Analysis of residual stresses in diamond films by x-ray diffraction and micro-raman spectroscopy," J. Appl. Phys., vol. 91, pp. $2466-2472,2002$

[25] S. Narayanan, S. R. Kalidindi, and L. S. Schadler, "Determination of unknown stress states in silicon wafers using microlaser raman spectroscopy,” J. Appl. Phys., vol. 82, pp. 2595-2602, 1997.

[26] G. H. Loechelt, N. G. Cave, and J. Menendez, "Polarized off-axis raman spectroscopy: A technique for measuring stress tensors in semiconductors," J. Appl. Phys., vol. 86, pp. 6164-6180, 1999.

[27] E. Anastassakis, "Strain characterization of polycrystalline diamond and silicon systems," J. Appl. Phys., vol. 86, pp. 249-257, 1999.

[28] V. Paillard, P. Puech, M. A. Laguna, P. Temple-Boyer, B. Caussat, J. P. Couderc, and B. De Maudit, "Resonant raman scattering in polycrystalline silicon thin films," Appl. Phys. Lett., vol. 73, pp. 1718-1720, 1998.

[29] D. Goustouridis, D. Tsoukalas, P. Normand, A. G. Kontos, Y. Raptis, and E. Anastassakis, "Parameters influencing the flatness and stability of capacitive pressure sensors fabricated with wafer bonding," Sens. Actuators, vol. A 76, pp. 403-408, 1999.

[30] M. Bowden, D. J. Gardiner, D. Wood, J. Burdess, A. Harris, and J. Hedley, "Raman and finite-element analysis of a mechanically strained silicon microstructure," J. Micromech. Microeng., vol. 11, pp. 7-12, 2001.

[31] G. Kaltas, A. G. Nassiopoulou, M. Siakavellas, and E. Anastassakis, "Stress effect on suspended polycrystalline silicon membranes fabricated by micromachining of porous silicon," Sens. Actuators, vol. A 68, pp. 429-434, 1998.

[32] M. S. Benrakkad, M. A. Benitez, J. Esteve, J. M. Lopez-Villegas, J. Samitier, and J. R. Morante, "Stress measurement by microraman spectroscopy of polycrystalline silicon structures," J. Micromech. Microeng., vol. 5, pp. 132-135, 1995.
[33] V. T. Srikar, A. K. Swan, B. B. Goldberg, M. S. Unlu, and S. M. Spearing, "Microscale stress measurement in a silicon flexure using raman spectroscopy," in Materials Research Society Symposium Proceedings, vol. 741, J9.5, 2003.

[34] M. Kayahara, Massachusetts Inst. Technol., private communication, 2001.

[35] F. R. Shanley, Strength of Materials. New York: McGraw-Hill, 1957.

[36] W. A. Brantley, "Calculated elastic constants for stress problems associated with semiconductor devices," J. Appl. Phys., vol. 44, pp. 534-535, 1973

[37] ABAQUS/CAE, Karlsson and Sorensen, Inc., Pawtucket, RI.

[38] J. F. Nye, Physical Properties of Crystals Clarendon, Oxford, U.K., 1985.

[39] Origin 6.1, Microcal Software, Inc., Northampton, MA

[40] H. J. McSkimin and P. Andreatch, "Elastic moduli of silicon vs. hydrostatic pressure at $25^{\circ} \mathrm{C}$ and $-195.8^{\circ} \mathrm{C}$," J. Appl. Phys., vol. 35, pp. 2161-2165, 1964.

[41] E. Bonera, M. Fanciulli, and D. N. Batchelder, "Raman spectroscopy for a micrometric and tensorial analysis of stress in silicon," Appl. Phys. Lett., vol. 81, pp. 3377-3379, 2002.

[42] D. J. Shim, V. T. Srikar, and S. M. Spearing, unpublished.

[43] L. A. Starman, J. A. Lott, M. S. Amer, W. D. Cowan, and J. S. Busbee, "Stress characterization of MEMS microbridges by micro-raman spectroscopy," Sens. Actuators, Phys. A, vol. 104, pp. 107-116, 2003.

[44] N. Tamura, R. S. Celestre, A. A. MacDowell, H. A. Padmore, R. Spolenak, B. C. Valek, N. M. Chang, A. Manceau, and J. R. Patel, "Submicron X-ray diffraction and its applications to problems in materials and environmental science," Rev. Sci. Instrum., vol. 73, pp. $1369-1372,2002$.

V. T. Srikar received the B.Tech. degree in metallurgical engineering from Banaras Hindu University, Varanasi, India, and the Ph.D. degree in materials science from the Massachusetts Institute of Technology (MIT), Cambridge, with a dissertation on the electromigration behavior and reliability of submicron aluminum interconnects in silicon integrated circuits.

Following postdoctoral appointments at MIT, he joined the Department of Mechanical Engineering at McGill University, Montreal, Canada, as an Assistant Professor with research interests in micro- and nanostructured materials and devices. His recent research activities include studies of the shock reliability of MEMS, identification of a novel mode of thermoelastic damping in polysilicon microresonators, self-assembly of nanoparticles on internal micromachined surfaces, and microscale stress measurement using spectroscopy. He is involved in the fluidic packaging of the MIT microrocket device and in the design and fabrication of a microfabricated solid-oxide fuel cell.

Anna K. Swan was born in Säffle, Sweden, in 1961. She received the Master's degree in physics engineering from Chalmers University, Gothenburg, Sweden, and the Ph.D. degree in physics at Boston University, Boston, MA, in 1994. Her dissertation topic was the spin-ordering on $\mathrm{NiO}(100)$ surfaces using metastable He* scattering, for which she received two student awards, the Nottingham prize and the Morton M. Traum Award.

She joined the Solid State Division at Oak Ridge National Laboratory as a Wigner Fellow. In 1999, she joined the Electrical and Computer Engineering Department at Boston University as a Research Assistant Professor. Her research topics are clustered around high-spatial resolution spectroscopy. Her active topics are spectral self interference as a means of improving fluorescence microscopy resolution, resonant Raman studies of single carbon nanotubes, and very sensitive and high spatial resolution stress-measurements in microelectromechanical systems (MEMS) using micro-Raman spectroscopy. 
M. Selim Ünlü (S'90-M'91-SM'95) was born in Sinop, Turkey, in 1964. He received the B.S. degree in electrical engineering from Middle East Technical University, Ankara, Turkey, in 1986 and the M.S.E.E. and Ph.D. degrees in electrical engineering from the University of Illinois, Urbana-Champaign, in 1988 and 1992, respectively. His dissertation topic dealt with resonant cavity enhanced (RCE) photodetectors and optoelectronic switches.

In 1992, he joined Boston University, as an Assistant Professor, and he is currently a Professor in the Department of Electrical and Computer Engineering. From January to July 2000, he worked as a Visiting Professor at University of Ulm, Germany. His career interest is in research and development of photonic materials, devices and systems focusing on the design, processing, characterization, and modeling of semiconductor optoelectronic devices, especially photodetectors. His current specific interests and expertise include high-speed RCE photodetectors, time and spatially resolved optical characterization of semiconductor materials, near-field and picosecond spectroscopy, near-field imaging of laser diodes, photonic bandgap and guided-wave devices, solid immersion lens microscopy, thermal imaging, biosensor fabrication and development of waveguide evanescent bio-imaging techniques, and hyperpolarized noble gas MRI He has authored and coauthored more than 150 technical articles and severa book chapters and magazine articles; edited one book; holds one U.S. patent; and has several patents pending.

Dr. Ünlü served as the Chair of IEEE Laser and Electro-Optics Society, Boston Chapter, winning the LEOS Chapter-of-the-Year Award, during 1994-1995. He served as the Vice President of SPIE New England Chapter from 1998 to 1999. He was awarded National Science Foundation Research Initiation Award in 1993, United Nations TOKTEN award in 1995 and 1996 and both the National Science Foundation CAREER and Office of Nava Research Young Investigator Awards in 1996. During 1999-2001, he served as the chair of the IEEE/LEOS technical subcommittee on photodetectors and imaging, and he is currently an Associate Editor for IEEE JOURNAL OF QUANTUM ELECTRONICS.
Bennett B. Goldberg was born in Boston, Mass. in 1959. He received the B.A degree from Harvard College, MA, in 1982 and the M.S. and Ph.D. degrees in physics from Brown University, Providence, RI, in 1984 and 1987, respectively.

Following a Bantrell Post-doctoral appointment at the Massachusetts Institute of Technology and the Francis Bitter National Magnet Lab, he joined the physics faculty at Boston University in 1989. Currently, he is a Professor of Physics and Professor of Electrical and Computer Engineering, and his active research interests include: near-field imaging of photonic bandgap, ring microcavity and single-mode waveguide devices; subsurface solid immersion microscopy for $\mathrm{Si}$ inspection; biosensor fabrication and development of waveguide evanescent bioimaging techniques; magnetooptics and magnetotransport of two- and one-dimensional electron systems; and nanooptics of interacting electron systems in quantum wells and quantum dot structures.

S. Mark Spearing received the Ph.D. degree from Cambridge University Engineering Department, Cambridge, U.K., in 1990.

From 1990 to 1992, he worked as a Research Engineer at the University of California at Santa Barbara, where he produced analytical models for the failure of high-temperature ceramic materials, and at Carborundum Microelectronics, where he was a member of the electronic packaging technical development team, from 1992 to 1994. He is an Associate Professor of Aeronautics and Astronautics at the Massachusetts Institute of Technology (MIT), Cambridge, where he has been since 1994. His technical interests include materials and structural analysis and design of MEMS, electronic packaging and advanced composites. Since 1995, he has been responsible for the materials and structures aspects of the design and packaging of the MIT MicroEngine, MicroRocket, Microchemical Power and Microhydraulic Transducer projects, as well as conducting cross-cutting underpinning technology development.

Dr. Spearing is a Member of the American Society of Mechanical Engineers (ASME) 